\title{
The tetrameric L27 domain complex as an organization platform for supramolecular assemblies
}

\author{
Wei Feng ${ }^{1,2}$, Jia-Fu Long ${ }^{1,2}$, Jing-Song Fan ${ }^{1}$, Tetsuya Suetake ${ }^{1} \&$ Mingjie Zhang ${ }^{1}$
}

L27 domain, initially identified in the Caenorhabditis elegans Lin-2 and Lin-7 proteins, is a protein interaction module that exists in a large family of scaffold proteins. The domain can function as an organization center of large protein assemblies required for establishment and maintenance of cell polarity. We have solved the high-resolution NMR structure of a tetrameric complex of L27 domains containing two SAP97-mLin-2 L27 domain heterodimers. Each L27 domain contains three $\alpha$-helices. The first two helices of each domain are packed together to form a four-helical bundle in the heterodimer. The third helix of each L27 domain forms another four-helical bundle that assembles the two heterodimers into a tetramer. The structure of the complex provides a mechanistic explanation for L27 domain-mediated polymerization of scaffold proteins, a process that is crucial for the assembly of supramolecular complexes in asymmetric cells.

\begin{abstract}
Clustering and asymmetric distribution of receptors, ion channels and associated protein complexes are essential to the polarity of neurons and epithelial cells. Such asymmetric targeting of large molecular assemblies is thought to be governed in part by modular scaffold proteins. L27 domain, initially identified in the C. elegans Lin-2 and Lin-7 proteins, is a protein interaction module that exists in a large family of scaffold proteins ${ }^{1}$. Formation of the trimeric Lin-2-Lin-7-Lin-10 complex requires L27 domains ${ }^{2,3}$ and has a central role in targeting receptor tyrosine kinase Let-23 signalsome to the basolateral surface of vulval precursor cells ${ }^{4,5}$. Mutations in Lin-2, Lin-7 or Lin-10 lead to a vulvaless phenotype, presumably by mistargeting of Let-23 (refs. 4,5). The mammalian orthologs of Lin-2, Lin-7 and Lin-10 are known as mammalian Lin-2 (mLin-2)/CASK, mLin-7/Velis/Mals and mLin10/X11 $\alpha /$ Mint1, respectively ${ }^{2,6-9}$. The mLin-2-mLin-7-mLin-10 tripartite complex has also been observed in the brain ${ }^{2,6}$, and this evolutionarily conserved protein complex has been implicated in the targeting of NMDA receptors and $\beta$-catenin assemblies to membrane subdomains in epithelia and neurons ${ }^{10,11}$.

A bioinformatics survey reveals that L27 domain-containing proteins are invariably scaffold proteins containing multiple proteinprotein interaction domains without intrinsic enzyme activities ${ }^{12}$. For example, SAP97 and mLin-2/CASK, which are members of a subset of membrane-associated guanylate kinases (MAGUKs), contain one and two L27 domains, respectively, in addition to their PDZ, SH3 and guanylate kinase-like domains. The N-terminal L27 domain of SAP97 can form specific heteromeric complexes with the N-terminal L27 domain of mLin-2, Dlg2 and Dlg3, respectively $3,13,14$. The C-terminal L27 domain of mLin-2 specifically binds to the L27 domain of mLin-7 (refs. 3,15). The discovery of L27 domains as specific protein-protein interaction modules capable of forming heteromeric complexes
\end{abstract}

suggests that L27 domains can integrate multiple scaffold proteins into supramolecular assemblies $13,13,15,16$. However, the molecular basis of the L27 domain-mediated protein assembly formation remains unclear. The structures of isolated L27 domains and their cognate heteromeric complexes are not known.

Here we show that the L27 domain of SAP97 and the N-terminal L27 domain of mLin-2 form a heterotetrameric complex. The structure of the SAP97-mLin-2 L27 complex was solved by NMR spectroscopy. The structure of the complex, together with data derived from various biochemical studies, establishes the roles of specific residues in the formation of the L27 heterotetrameric complex. The structure of the SAP97-mLin-2 L27 complex further suggests a mechanistic model for polymerization of L27 domain scaffold proteins.

\section{RESULTS}

L27 domains form specific heterotetrameric complexes

We first characterized the structural properties of the isolated L27 domains of SAP97 (referred to as L27S below), mLin-7, the $\mathrm{N}$-terminal L27 domain (referred to as $\mathrm{L} 27 \mathrm{~N}$ below), and the C-terminal L27 domain (referred to as L27C below) of mLin-2. Both NMR and CD studies revealed that the isolated L27N and L27C were largely unfolded and no stable homo-oligomers could be detected (Supplementary Fig. 1 online and data not shown). The relatively large chemical shift dispersion of the ${ }^{1} \mathrm{H},{ }^{15} \mathrm{~N}$ HSQC spectrum of the L27 domain of SAP97, together with a very broad elution peak on an analytical gel filtration column with an elution volume smaller than that for a theoretical monomeric protein, indicated that the individual domains of L27S can interact with each other (Supplementary Fig. 1 online and data not shown). However, both the broad resonances of the HSQC spectrum (Supplementary Fig. 1 online) and the high

${ }^{1}$ Department of Biochemistry, Molecular Neuroscience Center, The Hong Kong University of Science and Technology, Clear Water Bay, Kowloon, Hong Kong, China.

${ }^{2}$ These authors contributed equally to this work. Correspondence should be addressed to M.Z. (mzhang@ust.hk).

Published online 28 March 2004; doi:10.1038/nsmb751 
a

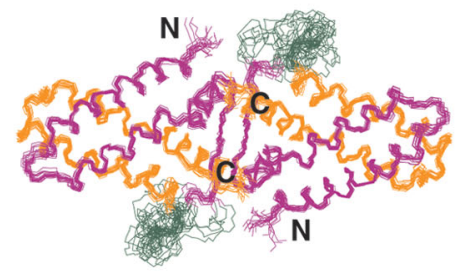

susceptibility to proteolytic degradation (with respect to the cognate hetero-L27 domain complex below) suggest that L27S does not form stable homo-oligomers. Consistent with earlier reports, mixing experiments using ${ }^{15} \mathrm{~N}$-labeled recombinant proteins showed that the L27S

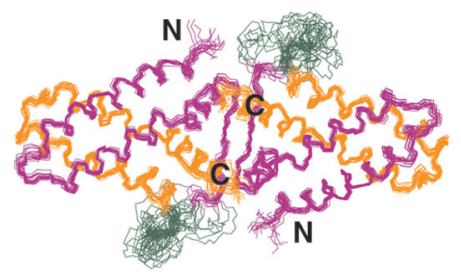

b
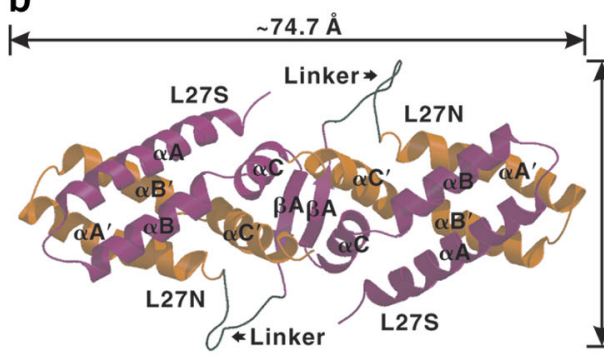

d

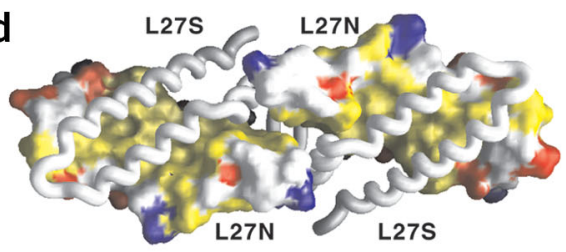

C

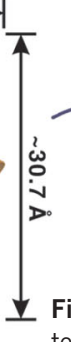

Figure 1 Structure of the SAP97-mLin-2 L27 tetramer complex. (a) Stereo view showing the backbone of 20 superimposed NMR-derived structures of the tetramer complex. L27S is purple, L27N of mLin-2 is gold and the flexible linker connecting L27S and L27N is dark green. (b) Ribbon diagram of a representative NMR structure of the tetramer complex, colored as in a. A prime after each secondary element (such as $\alpha A^{\prime}$ ) in L27N indicates the same secondary structure as in L27S. The flexible linker is indicated. (c) Ribbon diagram with

a structural comparison of L27S and L27N. (d) Surface representation of the packing interface of L27S-L27N. L27N is in surface representation, whereas L27S is in worm model. Hydrophobic residues are yellow, positively charged residues blue, negatively charged residues red and polar residues white. complex assembled into a tetramer with a molecular mass of $\sim 31.1 \mathrm{kDa}$ (Supplementary Fig. 2 online). Combined with the observation of the single set of the backbone amide resonances (Supplementary Fig. 1 online), we conclude that the L27S-L27N complex forms a symmetric dimer of dimers.

\section{Overall structure of the L27S-L27N complex}

Having established the mode of the assembly, we next determined the high-resolution NMR structure of the L27S-L27N tetramer using the single-chain fusion protein (Table 1). The use of this protein provided another advantage in the complex structural determination by NMR, as the L27S-L27N heterotetramer was 'decomposed' into a 'homodimer' consisting of two units of L27SL27N. Intersubunit NOEs between two units of L27S-L27N could be reliably identified using a ${ }^{13} \mathrm{C}$-edited $(\mathrm{F} 1),{ }^{13} \mathrm{C},{ }^{15} \mathrm{~N}$-filtered (F3) 3D NOESY spectrum on a ${ }^{13} \mathrm{C},{ }^{15} \mathrm{~N}$-labeled and nonlabeled protein mixture.

Other than the 4 residues from the C terminus of L27S (62-65), the 'glycineserine' covalent linker, and the N-terminal 13 residues of $\mathrm{L} 27 \mathrm{~N}$ (329-341) that are intrinsically flexible (that is, they lack any long-range NOEs and have substantially reduced ${ }^{1} \mathrm{H}_{-}{ }^{15} \mathrm{~N}$ heteronuclear NOEs, data not shown), the rest of the L27 complex is well defined of SAP97 and L27N of mLin-2 formed a stable heteromeric complex and that the $\mathrm{L} 27$ domain of mLin-7 and L27C of mLin-2 formed another heteromeric complex ${ }^{3,13-15}$ (data not shown). We chose the L27S-L27N pair for further structural analysis owing to the better spectral quality of this complex (Supplementary Fig. 1 online). The L27S-L27N heteromeric complex was obtained by coexpression of these two domains using a bicistronic plasmid. However, the L27S-L27N complex had limited stability and precipitated within $24 \mathrm{~h}$ at $25-30{ }^{\circ} \mathrm{C}$. Generation of a single-chain protein by fusing $\mathrm{L} 27 \mathrm{~N}$ to the $\mathrm{C}$ terminus of $\mathrm{L} 27 \mathrm{~S}$ yielded a protein with superior stability and homogeneity. The ${ }^{1} \mathrm{H},{ }^{15} \mathrm{~N}$ HSQC spectrum of the covalently linked protein is largely the same as that of the unlinked L27 complex (Supplementary Fig. 1 online). The regions that show observable chemical shift changes due to covalent linkage are limited to the $\mathrm{C}$ terminus of L27S and the N-terminal end of L27N. In addition, the backbone amides of residues 55-61 of L27S were extremely broad in the unlinked complex, and these residues becomes clearly observable in the covalently linked complex, presumably because of the covalent link-induced stabilization of this region (Supplementary Fig. 1 online). We conclude that the presence of the covalent linker has a minimal effect on the overall structure of the L27S-L27N complex. The L27S-L27N complexes, both with and without the covalent linker, eluted as single peaks from size-exclusion columns with a molecular mass corresponding to a tetramer (Supplementary Fig. 2 online). Analytical ultracentrifugation further confirmed that the L27S-L27N
(Fig. 1a). In each L27S-L27N heterodimer, the C terminus of L27S is in close proximity to the $\mathrm{N}$ terminus of L27N (Fig. 1a,b). Our structure is consistent with our earlier conclusion that the covalent linker does not alter the structure of the complex. The L27S-L27N tetramer forms a tightly packed, cylindrical structure. Each L27 domain contains three $\alpha$-helices $(\alpha \mathrm{A}-\alpha \mathrm{C})$ and L27S contains an additional $\beta$-strand $(\beta \mathrm{A})$ at the $\mathrm{C}$-terminal end. The $\alpha \mathrm{A}$ and $\alpha \mathrm{B}$ helices of each $\mathrm{L} 27$ domain form a helical hairpin. The two helical hairpins in each heterodimer are packed with each other to form a less common left-turn four-helical bundle at each end of the cylindrical tetramer ${ }^{17}$. A structural homology search using Dali (http://www.ebi.ac.uk/dali) revealed that the four-helical bundle formed by the $\alpha \mathrm{A}, \alpha \mathrm{B}, \alpha \mathrm{A}^{\prime}$ and $\alpha \mathrm{B}^{\prime}$ helices is similar to the four-helical bundle structures in many proteins including the de novo designed four-helical bundle protein s-824 (PDB entry 1P68) and a subdomain of guanine nucleotide-binding protein $\alpha-1$ subunit (PDB entry 1CIP). Each L27 domain contributes its third $\alpha$-helix to form an additional four-helical bundle at the center of the complex. The central helical bundle has an up-down-up-down topology with a marked right-handed twist when viewed from the top (Fig. 1b). One end of the central helical bundle is capped by a two-stranded antiparallel $\beta$-sheet formed by the $\beta$ A strands of two L27S domains. The central four-helical bundle is perpendicular to the two helical bundles at the ends of the complex (Fig. 1b).

Although L27S and L27N share only modest amino acid sequence identity (16.4\%), the structures of these two domains are remarkably similar (Fig. 1c). The structure-based sequence alignment shows that the loop connecting $\alpha \mathrm{A}$ with $\alpha \mathrm{B}$ is variable both in its length and amino acid composition (Fig. 2a). In contrast, the $\alpha \mathrm{B}$ and $\alpha \mathrm{C}$ helices of both L27 domains are connected with a single conserved amino acid. As a 
Figure 2 Detailed interactions between the L27 domains. (a) Structure-based sequence alignment of selected L27 domains. Extensive sequence analysis revealed that amino acid sequences of the L27 domains from the proteins that contain a single L27 domain can be best aligned with each other, and these L27 domains are categorized into the type A subfamily. Sequences of L27 domains from the proteins that contain two L27 domains connected in tandem can be aligned to each other and form another subfamily of L27 domains (type

B). The secondary structure of the rat SAP97 L27 domain (L27S) is at the top of the type A L27 domain sequences, and the secondary structure of the $\mathrm{N}$-terminal L27 domain of the rat $\mathrm{mLin}-2$ ( $L 27 \mathrm{~N})$ is at the top of the type B L27 domain sequences. Highly conserved hydrophobic residues are highlighted in yellow, other conserved residues in green. Blue asterisks, residues involved in the L27S-L27N heterodimer packing; turquoise dots, residues involved in packing of the central four-helical bundle of the tetramer; red asterisks, residues predicted to be critical for the mLin-7-mLin-2 L27-L27C (C-terminal L27 domain) heterodimer packing; triangles, single point mutations in $\mathrm{L} 27 \mathrm{~N}$ that disrupt the L27S-L27N complex formation. (b) Stereo view of the interactions of the four-helical bundle formed by $\alpha A$ and $\alpha B$ of L27S and $\alpha A^{\prime}$ and $\alpha B^{\prime}$ of $L 27 N$, with the side chains in the packing core in atom representation. (c) Stereo view of the interactions of the central four-helical bundle.

result, the $\alpha \mathrm{B}$ and $\alpha \mathrm{C}$ helices of the two L27 domains can be superimposed with very small deviation (r.m.s. deviation of 0.78 Å, Fig. 1c).

\section{L27S-L27N interactions in the heterodimer}

The packing of the four helices $\left(\alpha \mathrm{A}, \alpha \mathrm{B}, \alpha \mathrm{A}^{\prime}\right.$ and $\left.\alpha B^{\prime}\right)$ that stabilizes the L27S-L27N heterodimer is compact and extensive $\left(\sim 2,676 \AA^{2}\right.$ buried surface area in each four-helical bundle; Fig. 1d). The first two helices of both L27 domains are highly amphipathic, and the hydrophobic faces of the helices are packed with each other to form a large hydrophobic core, which presumably drives the heterodimer formation (Figs. 1d and 2). A kink at Ser353 in $\alpha \mathrm{A}^{\prime}$ of $\mathrm{L} 27 \mathrm{~N}$ ensures close packing of hydrophobic residues at the end of $\alpha A^{\prime}$ (Ile357 and Leu360) with hydrophobic amino acids in $\alpha A$ and $\alpha B$. Sequence alignment analysis reveals that the core-packing hydrophobic residues are highly conserved in L27 domains (Fig. 2a). We predict that the packing of other cognate pairs of the L27 domain heterodimers is also mediated by these key hydrophobic residues. In support of such proposed critical role of these conserved hydrophobic residues in the assembly of the complex, substitution of a single hydrophobic residue in the packing core with a neutral polar serine (such as L354S, L371S and L383S) led to the disruption of the L27S-L27N complex (Fig. 3a).

\section{Interface between the two L27S-L27N heterodimers}

The four helices that assemble the two L27S-L27N heterodimers form an up-and-down helical bundle (Fig. 2c). The packing force of the
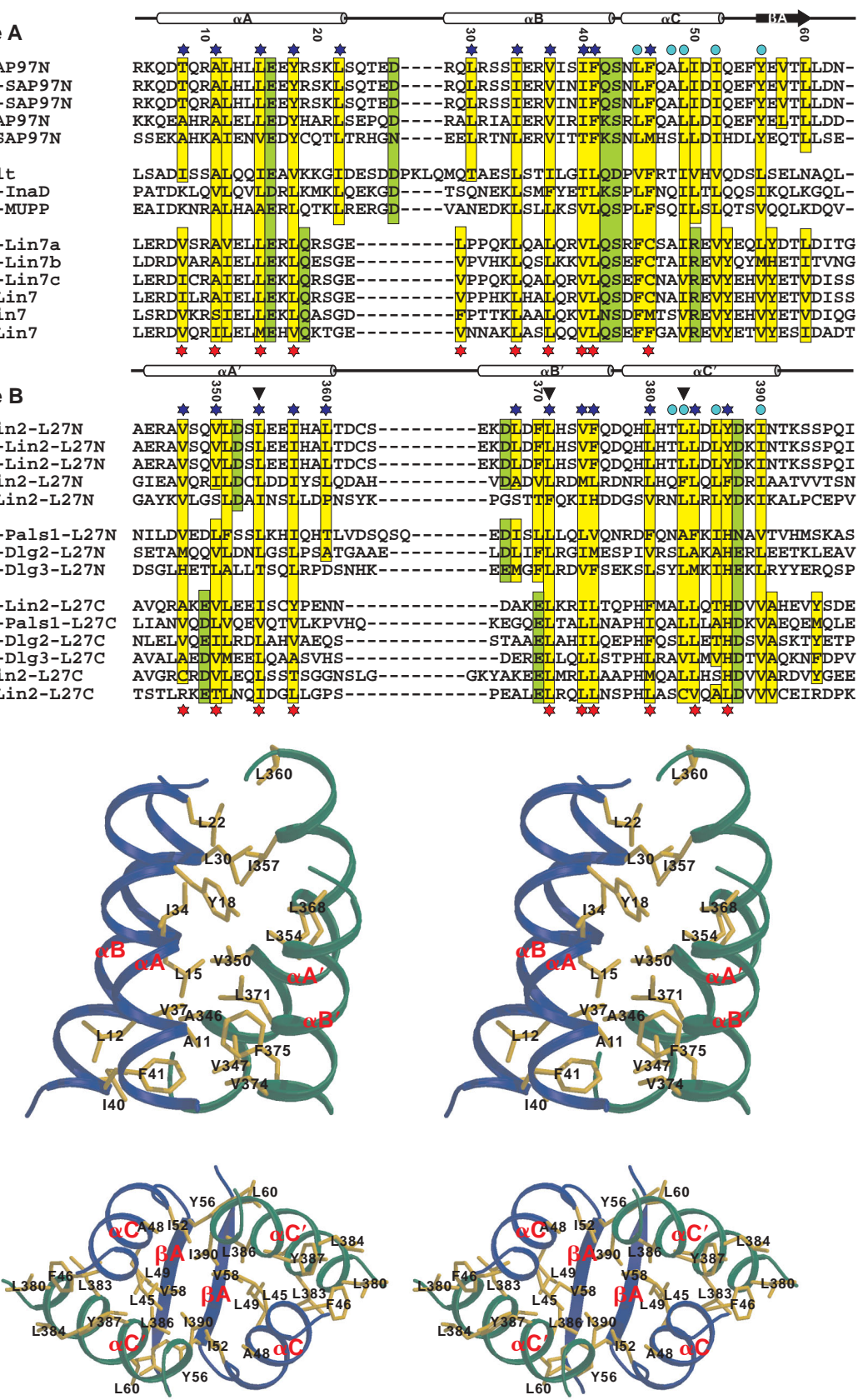

bundle is also hydrophobic, burying $\sim 2,081 \AA^{2}$ of surface area. Each $\alpha$-helix directly contacts two neighboring helices in the bundle. No direct interactions are observed between the two opposite $\alpha$-helices in the bundle (Fig. 2c). Consequently, the four-helical bundle contains a cavity ( $\sim 10 \AA$ in diameter) in its center, suggesting that this helical bundle is not as stable as the helical bundles formed within the L27S-L27N heterodimer (Fig. 2c).

The $\alpha \mathrm{C}$ helices of both $\mathrm{L} 27$ domains are considerably more hydrophobic than the $\alpha \mathrm{A}$ and $\alpha \mathrm{B}$ helices, and these hydrophobic residues are highly conserved (Fig. $2 \mathrm{a}$ ). In addition to the formation of the central helical bundle, the hydrophobic residues in the $\alpha \mathrm{C}$ helices also interact with hydrophobic residues from the helical bundles in each L27S-L27N dimer (Fig. 2a,c). We predict that the $\alpha \mathrm{C}$ helix-mediated dimer of the dimer assembly is a common feature in L27 domain-mediated complex formation. This prediction is 
a

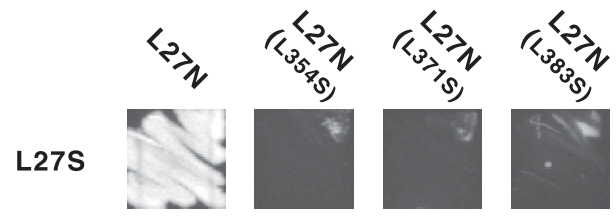

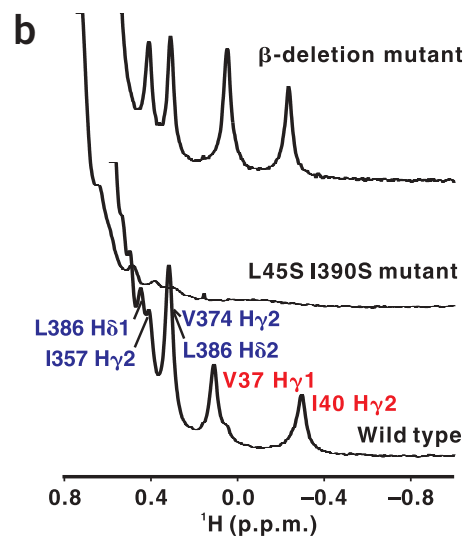

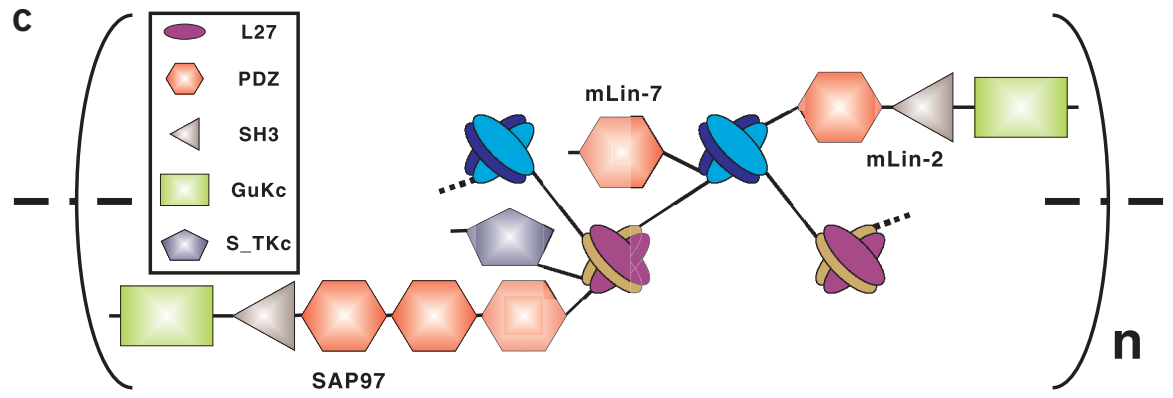

Figure 3 Dissection of the L27S-L27N complex. (a) Yeast two-hybrid assay. Mutation of individual conserved hydrophobic residues in the L27S-L27N heterodimer interface disrupts the L27 domain complex formation. Cotransfected yeast cells were selected by growth media lacking leucine, tryptophan, adenine and histidine. Switching the 'bait' and the 'prey' gave rise to the same interaction pattern (data not shown). (b) Structural determinants of the dimer-of-dimer interface of the L27S-L27N complex. The upfield-shifted ${ }^{1} \mathrm{H}$ NMR spectrum of the wild-type L27 complex shows characteristic peaks for residues in the interfaces between heterodimers (Val37 and Ile40 of L27S, and Val374 and Ile357 of L27N) as well as the dimer of dimers (Leu386). The L45S I390S mutant is devoid of any of these characteristic peaks, indicating that the protein is unfolded. In contrast, the $\beta$-strand deletion mutant showed essentially the same folded peaks in both the heterodimer and the dimer-of-dimer assemblies. (c) Schematic diagram showing L27 domainmediated polymerization of L27 domain scaffold proteins. The formation of large molecular assemblies is facilitated by the formation of a heterotetrameric complex between each cognate pair of L27 domains as well as by the existence of tandem L27 domains in the type B L27 domain scaffold proteins. In this model, the potential formation of polymerized protein complexes composed of SAP97, mLin-2 and mLin-7 is depicted.

supported by our finding that the complex formed between the mLin-7 L27 domain and the mLin-2 L27C domain also adopts a tetrameric structure (W.F., J.-F.L. and M.Z., unpublished results).

We tested our inference of the critical role of the central helical bundle in the L27S-L27N dimer formation and the assembly of the dimer of dimers from the complex structure using amino acid substitutions. The structure of the L27 domain complex suggests that replacing both Leu45 of L27S and Ile390 of L27N by hydrophilic but neutral serines would disrupt the hydrophobic packing of the central helical bundle (Fig. 2c). NMR analysis of the purified single-chain fusion protein containing these two point mutations showed that all the upfieldshifted methyl resonances, which are characteristic of both the heterodimer and the dimer-of-dimer assemblies in the wild-type protein, vanished (Fig. 3b). Our data indicate that such substitutions not only destroy the central helical bundle assembly, but also disrupt the formation of the heterodimer helical bundle. This finding also demonstrates that the formation of the dimer of dimers between the cognate L27 domain pairs is structurally indispensable for the L27 domainmediated protein assembly.
We further investigated the role of the $\beta$-strand in L27S in the assembly of the L27S-L27N complex by deleting the entire $\beta$-strand of the domain. We call this deletion mutant $\mathrm{L} 27 \mathrm{~S} \Delta \beta$. Analytical gel filtration chromatography showed that $\mathrm{L} 27 \mathrm{~S} \Delta \beta$ and L27N formed a complex with an elution volume indicative of a tetramer (Supplementary Fig. $2 c$ online). The NMR spectrum of the mutant complex showed that it was folded properly, with the central helical bundle assembled similarly to that of the wild-type complex (Fig. 3b). Taken together, these biochemical and structural data reveal that the antiparallel $\beta$-sheet capping one end of the central helical bundle is not essential for the L27 heteromeric complex assembly. Consistent with this conclusion, the amino acid sequences corresponding to the $\beta$-strand region of L27S in the different family members of the type A L27 domains are more divergent than the helical regions (Fig. 2a). Indeed, our preliminary NMR analyses reveal that some of the type A L27 domains do not contain a $\beta$-strand at their $\mathrm{C}$ termini (W.F., J.-F.L. and M.Z., unpublished results).

\section{DISCUSSION}

L27 domain proteins have increasingly been recognized as important organization centers for the polarized distribution of protein assemblies. The SAP97-mLin-2 L27 domain complex structure reported here provides a molecular basis for the interaction between cognate pairs of L27 domains. The identification of key residues with critical roles in the formation of the L27S-L27N heterodimer (and probably other pairs of L27 domain complexes) should be helpful in designing mutant L27 domain scaffold proteins to evaluate their functional roles in living cells.

A notable finding of this work is the discovery of the heterotetrameric assembly of the L27S-L27N complex. mLin-2 (in addition to other L27 domain-containing MAGUK proteins) contains two L27 domains arranged in tandem. Biochemical studies show that L27N does not physically interact with L27C (ref. 3, and W.F., J.-F.L. and M.Z., unpublished observations). Formation of the L27 domain complex between L27S and L27N would leave L27C to interact freely with the L27 domain of mLin-7. The dimer-of-dimer assembly of each cognate pair of L27 domains would therefore permit the SAP97mLin-2-mLin-7 scaffold proteins to polymerize into supramolecular complexes (Fig. 3c). This L27 domain-mediated polymerization model is consistent with experimental observations showing that L27 domain-containing scaffold proteins, such as SAP97 and mLin-2, often form large clusters $2,3,11,13,18,19$. During this L27 domainmediated scaffold protein assembly, other protein-binding domains (such as the PDZ and SH3 domains) would be free to recruit their specific target proteins (receptors, ion channels and their downstream partners). Thus, the structure of the L27 domain complex reveals that L27 domain-containing scaffold proteins are ideally suited for 
Table 1 Structural statistics for the family of 20 structures of the SAP97-mLin-2 L27 tetramer complex

\begin{tabular}{|c|c|}
\hline \multicolumn{2}{|l|}{ Distance restraints } \\
\hline Intraresidue $(i-j=0)$ & 2,294 \\
\hline Sequential $(|i-j|=1)$ & 1,144 \\
\hline Medium range $(2<|i-j|<4)$ & 1,120 \\
\hline Long range $(/ i-j />5)$ & 882 \\
\hline Intersubunit & 124 \\
\hline Hydrogen bonds & 264 \\
\hline Total & 5,828 \\
\hline \multicolumn{2}{|l|}{ Dihedral angle restraints } \\
\hline$\phi$ & 172 \\
\hline$\psi$ & 174 \\
\hline Total & 346 \\
\hline \multicolumn{2}{|c|}{ Mean r.m.s. deviations from experimental restraints } \\
\hline Distance $(\AA)$ & $0.020 \pm 0.000$ \\
\hline Dihedral angle $\left({ }^{\circ}\right)$ & $0.402 \pm 0.044$ \\
\hline \multicolumn{2}{|c|}{ Mean r.m.s. deviations from idealized covalent geometry } \\
\hline Bond $(\AA)$ & $0.002 \pm 0.000$ \\
\hline Angle $\left(^{\circ}\right)$ & $0.401 \pm 0.006$ \\
\hline Improper $\left({ }^{\circ}\right)$ & $0.303 \pm 0.007$ \\
\hline \multicolumn{2}{|l|}{ Mean energies $\left(\mathrm{kcal} \mathrm{mol}{ }^{-1}\right)^{\mathrm{a}}$} \\
\hline$E_{\mathrm{NOE}}$ & $121.47 \pm 5.31$ \\
\hline$E_{\text {cdih }}$ & $3.45 \pm 0.73$ \\
\hline$E_{\mathrm{L}-J}$ & $-704.57 \pm 21.59$ \\
\hline \multicolumn{2}{|l|}{ Ramachandran plot ${ }^{\mathrm{b}}$} \\
\hline \multicolumn{2}{|c|}{ Residues 4-63 in SAP97 and 339-394 in mLin-2 } \\
\hline Most favorable (\%) & 85.2 \\
\hline Additionally allowed (\%) & 11.9 \\
\hline Generously allowed (\%) & 2.5 \\
\hline \multicolumn{2}{|l|}{ Atomic r.m.s. differences ( $\AA$ ) } \\
\hline \multicolumn{2}{|c|}{ Full molecule (residues $4-61$ in SAP97 and 343-391 in mLin-2) } \\
\hline Backbone heavy atoms ( $N, C \alpha$ and $C^{\prime}$ ) & 0.48 \\
\hline Heavy atoms & 0.88 \\
\hline
\end{tabular}

None of the structures exhibits distance violations $>0.3 \AA$ or dihedral angle violations $>4^{\circ}$.

aThe final values of the square-well NOE and dihedral angle potentials were calculated with force constants of $50 \mathrm{kcal} \mathrm{mol}^{-1} \AA^{-1}$ and $200 \mathrm{kcal} \mathrm{mol}^{-1} \mathrm{rad}^{-1}$. bPROCHECK ${ }^{31}$ was used to assess the overall quality of the structures.

assembly of supramolecular signaling complexes. In these assemblies, L27 domain complexes act as nucleation centers for integrating scaffold proteins and their binding proteins into functional molecular circuits.

Any assemblies of multicomponent supramolecular complexes in living cells have to be dynamically regulated. L27 heterotetramers and potentially higher-order assemblies by multiple L27 domains are not exceptions. Nothing is yet known about the mechanism that regulates dynamic assembly of L27 domain complexes. Understanding the dynamic regulatory mechanism of L27 domain complexes is an important area of future research. The structure of the L27S-L27N tetramer shows that the central helical bundle contains a hydrophobic cavity with a diameter of $\sim 10 \AA$. It would be interesting to test whether low molecular-mass compounds could either weaken or strengthen the central helical bundle. Because the formation of the central helical bundle is essential for L27 heterotetramer formation, such compounds could be useful in manipulating the dynamic assembly of L27 domain complexes.

\section{METHODS}

Protein expression and purification. The single-chain fusion protein containing the L27 domain of rat SAP97 (residues 1-65) and L27N of rat
mLin-2/CASK (residues 329-400) connected with a glycine-serine linker was cloned into a modified version of pET32a vector ${ }^{20}$. Pro 2 and Pro64 of L27S and Pro334 of L27N were mutated to alanine to eliminate cis-trans isomerization of the prolines. All three prolines are within the unstructured linker region between L27S and L27N. Cys363 of L27N was changed to serine to prevent oxidization of the complex. Bacterial cells harboring the fusion protein expression plasmid were grown at $37^{\circ} \mathrm{C}$, and protein expression was induced by IPTG at the same temperature for $3 \mathrm{~h}$. Uniformly ${ }^{15} \mathrm{~N}$ - and ${ }^{15} \mathrm{~N}-{ }^{13} \mathrm{C}$-labeled proteins were prepared by growing bacteria in $\mathrm{M} 9$ medium containing ${ }^{15} \mathrm{NH}_{4} \mathrm{Cl}$ with or without ${ }^{13} \mathrm{C}_{6}$-glucose. The His-tagged, thioredoxin-containing protein was purified under native conditions using Ni-NTA agarose (Qiagen) affinity chromatography. After thrombin digestion, the $\mathrm{N}$-terminal His-tag and thioredoxin were removed by passing the digestion mixture through a DEAE-Sepharose column. The small amount of contaminant proteins was further removed from the target protein by size-exclusion chromatography.

NMR structure determination. NMR samples contained $\sim 1.5 \mathrm{mM}$ of the SAP97 mLin-2 L27-L27 domain complex in $100 \mathrm{mM}$ potassium phosphate, $\mathrm{pH} 6.5$, in $90 \% \mathrm{H}_{2} \mathrm{O} / 10 \% \mathrm{D}_{2} \mathrm{O}$ or $99.9 \% \mathrm{D}_{2} \mathrm{O}$. NMR spectra were acquired at $35^{\circ} \mathrm{C}$ on a Varian Inova $750 \mathrm{MHz}$ spectrometer equipped with an actively $z$-gradient-shielded triple-resonance probe. Sequential backbone and nonaromatic, nonexchangeable side chain resonance assignments of the protein were obtained by standard heteronuclear correlation experiments including HNCO, HNCA, HN(CO)CA, HNCACB, CBCA(CO)NH and HCCH-TOCSY, and confirmed by a 3D ${ }^{15} \mathrm{~N}$-separated NOESY experiment ${ }^{21,22}$. The side chains of the aromatics were assigned by ${ }^{1} \mathrm{H} 2 \mathrm{D}$ TOCSY-NOESY experiments of an unlabeled protein sample in $\mathrm{D}_{2} \mathrm{O}$. The stereospecific assignments of the valine and leucine methyl groups were obtained using a $10 \%{ }^{13} \mathrm{C}$-labeled sam$\mathrm{ple}^{23}$. Approximate interproton distance restraints were derived from NOESY spectra $\left(a^{1} \mathrm{H} 2 \mathrm{D}\right.$ homonuclear NOESY, a ${ }^{15} \mathrm{~N}$ separated-NOESY and a ${ }^{13} \mathrm{C}-$ separated NOESY). NOEs between two SAP97-mLin-2 L27 domain heterodimers were identified using a ${ }^{13} \mathrm{C}$-edited $(\mathrm{F} 1),{ }^{13} \mathrm{C},{ }^{15} \mathrm{~N}$-filtered (F3) 3D NOESY spectrum on a ${ }^{13} \mathrm{C},{ }^{15} \mathrm{~N}$-labeled and nonlabeled single-chain fusion protein mixture (1:1) (ref. 24). Intersubunit NOEs were concentrated in the central helical bundle region of the complex. No NOEs between $\alpha \mathrm{A}$ and $\alpha \mathrm{B}$ of L27S and $\alpha A^{\prime}$ and $\alpha B^{\prime}$ of L27N were observed in this double-filtered NOESY experiment. NOEs were grouped into three distance ranges: $1.8-2.7 \AA$ (1.8-2.9 $\AA$ for NOEs involving NH protons), $1.8-3.3 \AA$ (1.8-3.5 $\AA$ for NOEs involving $\mathrm{NH}$ protons) and $1.8-5.0 \AA$, corresponding to strong, medium and weak NOEs, respectively. Hydrogen bonding restraints (two per hydrogen bond where $r_{\mathrm{NH}-\mathrm{O}}=1.8-2.2 \AA$ and $r_{\mathrm{N}-\mathrm{O}}=2.2-3.3 \AA$ ) were generated from the standard secondary structure of the protein based on the NOE patterns and backbone secondary chemical shifts. Backbone dihedral angle restraints ( $\phi$ and $\psi$ angles) were derived from the secondary structure of the protein and backbone chemical shift analysis program TALOS ${ }^{25}$. Structures were calculated using $\mathrm{CNS}^{26}$. Figures were generated using $\mathrm{MolMol}^{27}, \mathrm{MolScript}^{28}$, Raster3 $\mathrm{D}^{29}$ and GRASP ${ }^{30}$.

Analytical ultracentrifugation. Sedimentation equilibrium measurements were made on a Beckman Optima XL-I equipped with an AN60 Ti 4-hole rotor at $20^{\circ} \mathrm{C}$. Sedimentation equilibrium data were collected at 16,000 r.p.m., $20,600 \mathrm{~g}$ at three different concentrations $(\sim 0.1,0.2$ and $0.3 \mathrm{mM})$ of protein. The proteins were dissolved in $100 \mathrm{mM}$ potassium phosphate buffer, $\mathrm{pH} 6.5$, containing $5 \mathrm{mM}$ DTT. The data were analyzed by a self-associating multimerization model using the manufacturer's software.

Gel filtration. Size-exclusion chromatography was carried out on an AKTA FPLC system using a Superose 12 10/30 column (Amersham Pharmacia Biotech). Protein samples $\left(\sim 2 \mathrm{mg} \mathrm{ml}^{-1}\right)$ were dissolved in $100 \mathrm{mM}$ potassium phosphate buffer containing $1 \mathrm{mM}$ DTT. The column was calibrated with the low-molecular-mass column calibration kit from Amersham Pharmacia Biotech.

Yeast two-hybrid assay. Yeast two-hybrid assays were done using the Matchmaker system 3 kit (Becton Dickinson Biosciences) according to the manufacturer's protocols. Briefly, L27S(1-65) of SAP97 and L27N(329-400) of mLin-2 (or its mutants) were subcloned into the pGBKT7 bait vector and the pGADT7 prey vector, respectively. Protein interactions in the yeast two-hybrid 
assays were monitored by cotransforming engineered plasmids into competent yeast (AH109) followed by plating onto selective media.

Coordinates. The atomic coordinates have been deposited in the Protein Data Bank (accession code 1RSO).

Note: Supplementary information is available on the Nature Structural \& Molecular Biology website.

\section{ACKNOWLEDGMENTS}

We thank D. Bredt for providing cDNAs of SAP97 and mLin-2 and for numerous insightful discussions, $\mathrm{H}$. Tochio and J. Tame for collecting ultracentrifugation data, M. Li for technical help and D. Banfield for comments on the manuscript. This work was supported by grants from the Research Grants Council of Hong Kong to M.Z. The NMR spectrometer used in this work was purchased with funds donated to the Biotechnology Research Institute by the Hong Kong Jockey Club. M.Z. is a Croucher Foundation senior research fellow.

\section{COMPETING INTERESTS STATEMENT}

The authors declare that they have no competing financial interests.

Received 7 January; accepted 25 February 2004

Published online at http://www.nature.com/natstructmolbiol/

1. Doerks, T. et al. L27, a novel heterodimerization domain in receptor targeting proteins Lin-2 and Lin-7. Trends Biochem. Sci. 25, 317-318 (2000).

2. Butz, S., Okamoto, M. \& Sudhof, T.C. A tripartite protein complex with the potential to couple synaptic vesicle exocytosis to cell adhesion in brain. Cel/ 94, 773-782 (1998).

3. Lee, S., Fan, S., Makarova, O., Straight, S. \& Margolis, B. A novel and conserved protein-protein interaction domain of mammalian Lin-2/CASK binds and recruits SAP97 to the lateral surface of epithelia. Mol. Cell. Biol. 22, 1778-1791 (2002).

4. Simske, J.S., Kaech, S.M., Harp, S.A. \& Kim, S.K. LET-23 receptor localization by the cell junction protein LIN-7 during C. elegans vulval induction. Cel/ 85, 195-204 (1996).

5. Kaech, S.M., Whitfield, C.W. \& Kim, S.K. The LIN-2/LIN-7/LIN-10 complex mediates basolateral membrane localization of the $C$. elegans EGF receptor LET-23 in vulva epithelial cells. Cel/ 94, 761-771 (1998).

6. Borg, J.P. et al. Identification of an evolutionarily conserved heterotrimeric protein complex involved in protein targeting. J. Biol. Chem. 273, 31633-31636 (1998).

7. Hata, Y., Butz, S. \& Sudhof, T.C. CASK: a novel dlg/PSD95 homolog with an N-terminal calmodulin-dependent protein kinase domain identified by interaction with neurexins. J. Neurosci. 16, 2488-2494 (1996).

8. Jo, K., Derin, R., Li, M. \& Bredt, D.S. Characterization of MALS/Velis-1, -2, and -3: a family of mammalian LIN-7 homologs enriched at brain synapses in association with the postsynaptic density-95/NMDA receptor postsynaptic complex. J. Neurosci. 19, 4189-4199 (1999).

9. Irie, M. et al. Isolation and characterization of mammalian homologues of Caenorhabditis elegans lin-7: localization at cell-cell junctions. Oncogene $\mathbf{1 8}$, 2811-2817 (1999).

10. Setou, M., Nakagawa, T., Seog, D.H. \& Hirokawa, N. Kinesin superfamily motor protein KIF17 and mLin-10 in NMDA receptor-containing vesicle transport. Science
288, 1796-1802 (2000).

11. Bamji, S.X. et al. Role of $\beta$-catenin in synaptic vesicle localization and presynaptic assembly. Neuron 40, 719-731 (2003).

12. Schultz, J., Milpetz, F., Bork, P. \& Ponting, C.P. SMART, a simple modular architecture research tool: identification of signaling domains. Proc. Natl. Acad. Sci. USA 95 , 5857-5864 (1998).

13. Chetkovich, D.M. et al. Postsynaptic targeting of alternative postsynaptic density-95 isoforms by distinct mechanisms. J. Neurosci. 22, 6415-6425 (2002).

14. Karnak, D., Lee, S. \& Margolis, B. Identification of multiple binding partners for the amino-terminal domain of synapse-associated protein 97. J. Biol. Chem. 277, 46730-46735 (2002).

15. Harris, B.Z., Venkatasubrahmanyam, S. \& Lim, W.A. Coordinated folding and association of the LIN-2, 7 (L27) domain: An obligate heterodimerization module involved in assembly of signaling and cell polarity complexes. J. Biol. Chem. 277 34902-34908 (2002).

16. Roh, M.H. et al. The Maguk protein, Pals1, functions as an adapter, linking mammalian homologues of Crumbs and Discs Lost. J. Cell Biol. 157, 161-172 (2002).

17. Richardson, J.S. \& Richardson, D.C. In Prediction of Protein Structure and Principles of Protein Conformation. (ed. Fasman, G.) 1-98 (Plenum, New York, 1989).

18. Wu, H., Reuver, S., Kuhlendahl, S., Chung, W. \& Garner, C. Subcellular targeting and cytoskeletal attachment of SAP97 to the epithelial lateral membrane. J. Cell Sci. 111, 2365-2376 (1998).

19. Hsueh, Y.-P. et al. Direct interaction of CASK/LIN-2 and syndecan heparan sulfate proteoglycan and their overlapping distribution in neuronal synapses. J. Cell Biol. 142, 139-151 (1998).

20. Feng, W., Fan, J.S., Jiang, M., Shi, Y.W. \& Zhang, M. PDZ7 of glutamate receptor interacting protein binds to its target via a novel hydrophobic surface area. J. Biol. Chem. 277, 41140-41146 (2002).

21. Clore, G.M. \& Gronenborn, A.M. Determining the structures of large proteins and protein complexes by NMR. Trends Biotechnol. 16, 22-34 (1998).

22. Kay, L.E. \& Gardner, K.H. Solution NMR spectroscopy beyond 25 kDa. Curr. Opin. Struct. Biol. 7, 722-731 (1997).

23. Neri, D., Szyperski, T., Otting, G., Senn, H. \& Wuthrich, K. Stereospecific nuclear magnetic resonance assignments of the methyl groups of valine and leucine in the DNA-binding domain of the 434 repressor by biosynthetically directed fractional ${ }^{13} \mathrm{C}$ labeling. Biochemistry 28, 7510-7516 (1989).

24. Zwahlen, C. et al. Methods for measurement of intermolecular NOEs by multinuclear NMR spectroscopy: application to a bacteriophage N-peptide/boxB RNA complex. J. Am. Chem. Soc. 119, 6711-6721 (1997).

25. Cornilescu, G., Delaglio, F. \& Bax, A. Protein backbone angle restraints from searching a database for chemical shift and sequence homology. J. Biomol. NMR 13, 289-302 (1999).

26. Brunger, A.T. et al. Crystallography \& NMR system: a new software suite for macromolecular structure determination. Acta Crystallogr. D 54, 905-921 (1998).

27. Koradi, R., Billeter, M. \& Wuthrich, K. MOLMOL: a program for display and analysis of macromolecular structures. J. Mol. Graph. 14, 51-55 (1996).

28. Kraulis, P.J. MOLSCRIPT: a program to produce both detailed and schematic plots of protein structures. J. Appl. Crystallogr. 24, 946-950 (1991).

29. Merritt, E. \& Murphy, M. Raster3D version 2.0: a program for photorealistic molecular graphics. Acta Crystallogr. D 50, 869-873 (1994).

30. Nicholls, A., Sharp, K. \& Honig, B. Protein folding and association-insights from the interfacial and thermodynamic properties of hydrocarbons. Proteins 11, 281-296 (1991).

31. Laskowski, R.A., Rullman, J.A., MacArthur, M.W., Kaptein, R. \& Thornton, J.M. AQUA and PROCHECK-NMR: programs for checking the quality of protein structures solved by NMR. J. Biomol. NMR 8, 477-886 (1996). 